\title{
Re: The role of ENT surgeons in snoring assessment: some prospective preliminary evidence
}

Sir,

We read with interest the paper by Robertson et al. ${ }^{1}$ which outlines a proposed future role of ENT surgeons in assessing patients with snoring. This case-series (rather than a cohort study) showed a significant correlation between hard nasal symptoms and positive clinical examination of the nose in snorers, which is not surprising. The role of ENT surgeons is very important in assessing snoring. Being ENT surgeons does not exclude the fact that we are physicians who operate on a proportion of patients who come to clinic. Endoscopic examination is important in order to assess the level of snoring as well as exclude any structural abnormality in the upper aerdigestive tract, even with the absence of any nasal symptoms or finding. Traditionally, many procedures have been advocated to help this purpose, such as Mueller's manoeuvre and sleep endoscopy.

Full assessment of snoring should involve general and local factors which contribute to the patient's complaint, such as any history of apnoea attacks, high body mass index, reflux, smoking, alcohol consumption, uvula size and laxity of soft palate, collar size and base of tongue. The paper did not clearly identify the potential impor- tance of these factors. Univariate analysis is not the appropriate test for this study because it is used in parametric data however the data in this study is non-parametric.

Finally, we disagree with the authors in handing over snoring assessment to other allied health professionals, which may affect training.

\author{
Shayah, A. \& Coatesworth, A.P. \\ Department of ENT, York Hospital, \\ Wigginton Road, York, \\ North Yorkshire, YO31 8HE, UK. \\ Email: assemshayah@aol.com
}

\section{Conflict of interest}

None to declare.

\section{Reference}

1 Robertson S., Young D., McGarry G.W. et al. (2008) The role of ENT surgeons in snoring assessment: some prospective prelimin ary evidence. Clin. Otolaryngol. 33, 458461

\section{Response to Shayah and Coatesworth}

Sir,

We thank Shayah \& Coatesworth for their interest in our paper. We agree that ENT surgeons are, indeed, doctors who operate on a proportion of patients attending outpatient clinics. As reported in our paper, 12 of 93 snorers $(13 \%)$ attending our clinic underwent surgical intervention following assessment. We have clearly demonstrated that endoscopic examination of snorers is unnecessary in the absence of specific symptoms. Shayah and Coatesworth cite no clinical evidence to support their contradictory claim.

We have commented on the demise of sleep nasoendoscopy in paragraph 1 of our paper and do not feel that further comment is necessary. Whilst the Muller manoeuvre may form part of 'traditional' snoring assessment in some ENT centres, there is an overwhelming lack of good clinical evidence to support the reproducibility, clinical relevance and positive predictive value of this procedure. There is also a lack of good clinical evidence to support the subjective measurement of uvula size, tongue base bulk and soft-palate laxity.

The potential importance of general health factors which may contribute to snoring is not disputed. Body mass index, smoking history and alcohol consumption were measured for all patients in our study and appropriate advice given by the principal author. Such health 